\title{
Telessaúde Sergipe: principais demandas dos profissionais da equipe de saúde bucal
}

\author{
Telessaúde Sergipe: main demands of the professionals of the oral health \\ Telessaúde Sergipe: principales demandas de los profesionales del equipo de salud bucal
}

Recebido: 26/02/2021 | Revisado: 05/03/2021 | Aceito: 08/03/2021 | Publicado: 16/03/2021

\author{
Graziane Ribeiro Couto \\ ORCID: https://orcid.org/0000-0002-4443-7867 \\ Universidade Federal de Sergipe, Brasil \\ E-mail: grazianeribeiro.couto@gmail.com \\ Natalli Ingreth Aparecida dos Santos \\ ORCID: https://orcid.org/0000-0003-0131-8144 \\ Universidade Federal de Sergipe, Brasil \\ E-mail: natalliingreth@gmail.com \\ Marcos Antônio Lima dos Santos \\ ORCID: https://orcid.org/0000-0002-7589-4809 \\ Universidade Federal de Sergipe, Brasil \\ E-mail: marcosals@outlook \\ Eneida Carvalho Gomes Ferreira \\ ORCID: https://orcid.org/0000-0001-9761-2879 \\ Fundação Estadual de Saúde do Estado de Sergipe, Brasil \\ E-mail: eneida.learning@gmal.com \\ Regiane Cristina do Amaral \\ ORCID: https://orcid.org/0000-0002-9191-0960 \\ Universidade Federal de Sergipe, Brasil \\ E-mail: amaralre@yahoo.com.br
}

\begin{abstract}
Resumo
O início da implantação do Programa Teleodontologia no Estado de Sergipe aconteceu em 2012 em parceria com o Ministério da Saúde, contemplando os 75 municípios sergipanos com pontos de acesso presentes em unidades e clínicas de Atenção Primária à Saúde. Atualmente, o Núcleo de Teleodontologia Sergipe contempla 100\% dos municípios do Estado com 163 pontos implantados. Assim, o presente estudo teve por objetivo identificar as demandas enviadas pelos cirurgiões-dentistas do Estado de Sergipe ao Telessaúde no período de 2013 a 2019. Os dados foram coletados de bancos de dados da Teleodontologia, contendo informações de 230 profissionais cadastrados, totalizando uma amostra de 137 dúvidas. Dentre as dúvidas, aproximadamente $30 \%$ são sobre diagnóstico, seguidas por $13 \%$ sobre tipo de medicação principalmente relacionada a grupos como gestantes e hipertensos. Dentre os municípios que mais utilizam a ferramenta tem-se Aracaju e Nossa Senhora do Socorro. Observa-se uso gradativo por parte dos profissionais, sendo no ano de 2013 a 2014 com apenas 27 perguntas. Houve grande adesão pelo uso da ferramenta pelos profissionais auxiliando no processo de melhor atendimento à população e diagnósticos pautados em referencias do Ministério da Saúde.
\end{abstract}

Palavras-chave: Telemonitoramento; Teleodontologia; Odontologia; Atenção primária à saúde.

\begin{abstract}
The implementation of the Teleodontology Program in the State of Sergipe began in 2012 in partnership with the Ministry of Health, covering the 75 municipalities in Sergipe with access points present in Primary Health Care units and clinics. Currently, the Sergipe Teleodontology Center contemplates $100 \%$ of the state's municipalities with 163 points in place. Thus, the present study aimed to identify the demands sent by dental surgeons from the State of Sergipe to Telehealth in the period from 2013 to 2019. Data werecollected from Teleodontology databases, containing information from 230 registered professionals, totaling a sample. of 137 doubts. Among the doubts, approximately $30 \%$ are about diagnosis, followed by $13 \%$ about the type of medication, mainly related to groups such as pregnant women and hypertensive patients. Among the municipalities that use the tool, the most are Aracaju and Nossa Senhora do Socorro. There is a gradual use by professionals, being in the year 2013 to 2014 with only 27 questions. There was great adherence by the use of the tool by professionals, assisting in the process of better service to the population and diagnoses based on references from the Ministry of Health.
\end{abstract}

Keywords: Telemonitoring; Teleodontology; Dentistry; Primary health care.

\section{Resumen}

La implementación del Programa de Teleodontología en el Estado de Sergipe se inició en 2012 en alianza con el Ministerio de Salud, abarcando los 75 municipios de Sergipe con puntos de acceso presentes en unidades y clínicas de Atención Primaria de Salud. Actualmente, el Centro de Teleodontología de Sergipe contempla el 100\% de municipios 
del estado con 163 puntos en su lugar. Así, el presente estudio tuvo como objetivo identificar las demandas enviadas por los cirujanos dentistas del Estado de Sergipe a Telesalud en el período de 2013 a 2019. Se recogieron datos de las bases de datos de Teleodontología, que contienen información de 230 profesionales registrados, totalizando una muestra. De 137 dudas. . Entre las dudas, aproximadamente un 30\% son sobre diagnóstico, seguido de un 13\% sobre el tipo de medicación, principalmente relacionadas con grupos como mujeres embarazadas e hipertensos. Entre los municipios que más utilizan la herramienta se encuentran Aracaju y Nossa Senhora do Socorro. Hay un uso paulatino por parte de los profesionales, estando en el año 2013 al 2014 con solo 27 preguntas. Hubo gran adherencia por el uso de la herramienta por parte de los profesionales, asistiendo en el proceso de mejor servicio a la población y diagnósticos basados en referencias del Ministerio de Salud.

Palabras clave: Telemonitorización; Teleodontología; Odontología; Primeros auxilios.

\section{Introdução}

O sistema de saúde bucal público brasileiro passou por momentos de cunho predominantemente curativo e excludente, com os modelos Incremental no ano de 1950 e Simplificado no período de 1970, com uma abordagem individual que tinha como alvo principal a população em idade escolar, tida como epidemiologicamente mais vulnerável e, ao mesmo tempo, a mais sensível às intervenções de saúde pública (Narval, 2016). No ano de 1990, com o advento do Sistema Único de Saúde (SUS) e posteriormente com a criação do Programa Saúde da Família (PSF) e com a incorporação do profissional cirurgião-dentista (ano de 2000), esse cenário passou por modificações, visto que se deu ênfase a tratamentos preventivos em saúde, que passaram a ser de livre acesso a todos os grupos populacionais (DAB, 2019).

Contudo, o principal alavanque em relação à saúde bucal deu-se pelo Programa Brasil Sorridente (2004), que teve como objetivos reorganizar o serviço na Atenção Primária à Saúde (APS) e criar assistência na Atenção Secundária em Saúde (Brasil, 2019a). O Programa Brasil Sorridente, por meio da reorganização da APS, colocou entre os atendimentos grupos prioritários, como gestantes, idosos, crianças, entre outros, pacientes que muitas vezes requerem um cuidado especial por parte do profissional (Brasil, 2019a).

Na tentativa de auxiliar os profissionais de saúde, foi criado em 2007 o Programa Nacional Telessaúde Brasil Redes (Brasil, 2019b), com o intuito de fortalecer e melhorar a qualidade do atendimento da APS no Sistema Único de Saúde (SUS), integrando Educação Permanente em Saúde (EPS) e apoio assistencial por meio de ferramentas e tecnologias da informação e comunicação. Esse instrumento é constituído por Núcleos Estaduais, Intermunicipais e Regional, que desenvolvem e ofertam serviços específicos para profissionais e trabalhadores do SUS, dentre eles teleconsultoria, segunda opinião formativa, teleducação e oferta nacional de telediagnóstico (Brasil, 2019b).

A teleconsultoria consiste em esclarecimento de dúvidas sobre procedimentos clínicos, ações de saúde e questões relativas a processo de trabalho no formato de pergunta e resposta entre profissionais de saúde. Esse sistema pode ser de forma síncrona - realizada em tempo real, geralmente por chat, webconferência, videoconferência ou serviço telefônico; ou assíncrona - realizada por meio de mensagens off-line que devem ser respondidas em até 72h. A segunda opinião formativa trata-se de resposta sistematizada, construída com base em revisão bibliográfica e evidências científicas e clínicas a perguntas originadas das teleconsultorias. Por sua vez, a teleducação consiste em atividades educacionais à distância por meio de tecnologias de informação e comunicação para apoiar a qualificação de estudantes, profissionais e trabalhadores da área da saúde. A oferta nacional de telediagnóstico se configura em ampliação da possibilidade de realização de exames com emissão de laudo à distância, diminuindo custos com deslocamento de pacientes, aumentando a resolubilidade da Atenção Básica e, ainda, ampliando a oferta em especialidades (Brasil, 2019b; Brasil, 2020a).

O início da implantação do programa no Estado de Sergipe aconteceu em 2012 em parceria com o Ministério da Saúde, contemplando os 75 municípios sergipanos com pontos de acesso presentes em unidades e clínicas de Atenção Primária à Saúde. 
Esse programa tem como objetivo qualificar os profissionais da APS utilizando tecnologias da informação e comunicação e acesso à Educação Permanente em Saúde. Esses profissionais podem fazer teleconsultas com especialistas que estão no Núcleo de Telessaúde Sergipe. Essa ferramenta de comunicação permite que realizem consultas, esclareçam dúvidas sobre procedimentos clínicos e ações de saúde, como também tenham uma segunda opinião formativa (Sergipe, 2019).

O Núcleo de Telessaúde Sergipe tem sua gestão realizada pela Secretaria de Estado da Saúde e operacionalização através da FUNESA, tendo para isso como parceira a Universidade Federal de Sergipe (UFS). Com o escopo de garantir o processo de qualificação à distância, conta com uma equipe formada por especialistas, mestres e doutores com experiência na APS e em tecnologia da informação (Brasil, 2019c). Dentre as funções que compõem o Núcleo Telessaúde há teleconsultores (enfermeiro, médico e odontólogo), médico telerregulador, monitor de campo, analistas e técnicos em informática, referência técnica em Saúde Pública e Coletiva, além de assistentes administrativos e analistas educacionais (Brasil, 2019c).

No ano de 2019, o Núcleo de Telessaúde Sergipe contemplava 100\% dos municípios do Estado com 163 pontos implantados. O Estado de Sergipe tem 490 equipes de Estratégias de Saúde da Família com cobertura em saúde bucal, alcançando 76,9\% da população, segundo o relatório de dezembro de 2019 (Brasil, 2020b). O Núcleo Telessaúde tem uma subdivisão denominada Teleodontologia, que trata de dúvidas voltadas para o cuidado em saúde bucal. Neste estudo, tais dúvidas foram agrupadas por categorias apresentadas com maior frequência.

Diante do exposto, o objetivo do presente estudo foi identificar quais as principais demandas dos cirurgiõesdentistas do Estado de Sergipe demandaram ao Telessaúde.

\section{Metodologia}

Esta pesquisa foi aprovada através do Certificado de Apresentação de Apreciação Ética (CAAE) 22983819.5.0000.5546 pelo Comitê de Ética em Pesquisa da Universidade Federal de Sergipe.

Trata-se de um estudo transversal, com dados extraídos do Programa Telessaúde do Estado de Sergipe, vinculados aos registros odontológicos dos anos de 2013 a 2019, obtidos junto à Secretaria do Estado de Sergipe por meio da Fundação Estadual de Saúde (FUNESA).

Dentre os dados da planilha do Telessaúde tem-se ano, local e dúvida da Equipe de Saúde Bucal (ESB), que por questões éticas, visto que em alguns municípios há apenas um profissional, estes dados não foram apresentados. Foram coletados todos os dados de dúvidas dos profissionais que enviaram para o Telessaúde no período analisado. Assim o tamanho amostral foi composto por todas as dúvidas apresentadas.

A análise dos dados foi realizada de forma descritiva utilizando-se a ferramenta Microsoft Excel versão 2007, sendo agrupado as perguntas que são de forma descritiva por assuntos principais.

\section{Resultados e Discussão}

O Estado de Sergipe contava em 2019 com 230 profissionais cirurgiões dentista cadastrados no SUS. Destes, o programa Telessaúde recebeu no período de 2013 a 2019, 137 dúvidas, as quais foram categorizadas de acordo com o tema principal do questionamento, conforme detalhado na Tabela 1. 
Tabela 1 - Dúvidas dos cirurgiões-dentistas cadastrados no Programa Telessaúde do Estado de Sergipe, $2013-2019$.

\begin{tabular}{lll}
\hline Dúvidas & $\mathbf{n}$ & \% \\
\hline Diagnóstico & 21 & 29,9 \\
Medicação & 17 & 12,4 \\
Material de apoio & 16 & 11,7 \\
Encaminhamento & 11 & 8 \\
Gestante & 9 & 6,6 \\
Criança & 7 & 5,1 \\
Procedimentos preventivos & 7 & 5,1 \\
Esterilização & 6 & 4,4 \\
Procedimentos cirúrgicos & 6 & 4,4 \\
Contenção de riscos & 4 & 2,9 \\
Idoso & 3 & 2,2 \\
Materiais dentários & 3 & 2,2 \\
Procedimentos administrativos & 3 & 2,2 \\
Recém-nascido & 2 & 1,5 \\
Visita domiciliar & $\mathbf{1 3 7}$ & 1,5 \\
\hline Total & 3 & \\
\hline & & \\
\hline
\end{tabular}

Fonte: Coleta de dados - material cedido pela coordenação da Fundação do Estado de Sergipe.

Entre as dúvidas, as que aparecem com maior prevalência são as de diagnóstico e medicação, sendo elas:

- "Paciente de 55 anos, usuária de próteses totais superiores mal adaptadas há mais de 10 anos, compareceu à UBS apresentando lesão na mucosa lateral da bochecha (lado esquerdo) há mais ou menos um ano. A lesão apresenta-se pedunculada, com coloração igual à da mucosa, sem sangramento, sem sintomatologia dolorosa. Qual o possivel diagnóstico?”.

- $\quad$ "Paciente de 53 anos, diabético descompensado (glicoteste 352), hipertenso (PA 14 por 9 mm Hg), reclama de dor na região das unidades 36, 37, 38. Com drenagem purulenta espontânea e mobilidade dos molares. Quadro de febre, tratei como periodontite, mediquei com amoxicilina e metronidazol. Solicitei radiografia periapical... Quais as minhas próximas condutas se na radiografia revelar uma lesão maior? Solicito panorâmica ou já biópsia?”.

- "Paciente do gênero feminino chegou ao consultório odontológico queixando-se de uma lesão branca na área de palato mole, próximo aos molares superiores, lado esquerdo, indolor, apareceu há mais ou menos uma semana, não cedeu à raspagem. Podemos estar diante de um quadro de paciente com papiloma vírus?”.

- "Qual a verdadeira interferência que os antibióticos podem trazer à dentição decídua e permanente quando usados por recém-nascidos?”.

- "Quais os riscos que os antibióticos podem trazer à dentição decídua e permanente da criança quando usados por gestantes?". 
- “Como atuar em um paciente que está com dor dente tipo uma pulpite e é diabdetico com insuficiencia renal, faz diálice peritonial e está com a PA (pressão arterial) muita alta, entrei em contato com o médico da área que conseguiu baixar a PA mas a glicemia não?

A terceira maior demanda por parte dos profissionais foi relacionada a material de apoio para se guiar diante de uma situação clínica, tais como:

- "Qual o material mais indicado para se utilizar nas pulpotomias realizadas nas UBS, pois apenas dispomos de hidróxido de cálcio e anestésico. Podemos utilizar esse material ou seria recomendado a pasta guedes? Como fazer a pasta Guedes?"

- "Gostaria de saber de em Sergipe existe referência para pacientes com síndrome de Menkes gostaria de materiais relacionados ao assunto citado”.

O programa de Teleodontologia é uma ferramenta importante, visto que permite aos profissionais de lugares remotos a terem acesso a informação, auxiliando na tomada de decisões para um melhor tratamento e, consequentemente, melhorando o acesso à saúde bucal e diminuindo custos (Jampani, Nutalapati, Dontula, \& Boyapati, 2011). No presente estudo verifica-se que há uma gradativa adesão dos profissionais pelo uso do Telessaúde, visto que somente em 2019 há $100 \%$ de cobertura das Unidades de Saúde no Estado, isso é observado quando no período de 2013 a 2014 há apenas 27 perguntas dos profissionais cirurgiões dentistas, sendo este número aumentado gradativamente ao longo dos anos.

Muitos dos dentistas que participaram do Telessaúde do Estado de Sergipe estão localizados distantes dos grandes centros, às vezes em áreas rurais, em que até mesmo o encaminhamento desses pacientes se torna difícil. Portanto, pela própria dificuldade de acesso, o Telessaúde, por ser uma ferramenta virtual, que auxilia o profissional na tomada de decisão (Tabela 2).

Tabela 2 - Municípios que mais apresentaram dúvidas no Programa Telessaúde do Estado de Sergipe, 2013 -2019.

\begin{tabular}{lr}
\hline Município & Perguntas enviadas \\
\hline Aracaju & 20 \\
Nossa Senhora do Socorro & 14 \\
Divina Pastora & 9 \\
Siriri & 5 \\
Nossa Senhora das Dores & 4 \\
Canhoba & 3 \\
Ribeirópolis & 3 \\
Rosario Do Catete & 3 \\
\hline
\end{tabular}

Fonte: Coleta de dados - material cedido pela coordenação da Fundação do Estado de Sergipe.

Nos dados apresentados do presente estudo verifica-se que a maior parte das perguntas foi enviada pelos profissionais da capital (Aracaju), muito provável porque na capital se reúne a maior parte dos profissionais cirurgiões dentista cadastrados no Estado. Contudo, é verificado que o município de Nossa Senhora do Socorro distando 18 km da capital, Divina Pastora distando $36 \mathrm{~km}$ da capital, Siriri distando $55 \mathrm{~km}$ da capital e Canhoba distando $150 \mathrm{~km}$ da capital contam com dúvidas.

O profissional que atua junto ao Telessaúde tem até 72 horas para responder em formato de texto as dúvidas dos profissionais, através de uma plataforma de tecnologia à distância e estas respostas são pautadas em evidências cientificas tendo como base referências atualizadas, o site da Ministério da Saúde e da BIREME (FUNESA, 2021). 
Correia et al. (2014) relataram as experiências desenvolvidas no Núcleo Técnico-Científico do Programa Nacional Telessaúde Brasil Redes no Mato Grosso do Sul, no qual foram cadastrados no Sistema de Teleconsultorias 2.317 profissionais de saúde, sendo 222 cirurgiões-dentistas e 81 auxiliares de saúde bucal. Quanto às teleconsultorias assíncronas, houve 368 solicitações, das quais 6,79\% foram de cirurgiões-dentistas. Foram geradas 579 respostas das 368 teleconsultorias no sistema, e desse total $61(10,53 \%)$ foram de Teleodontologia.

Muitas vezes, como se observa no presente estudo, o cirurgião-dentista clínico da APS não conhece os diagnósticos de lesões bucais, que certamente são da alçada de especialistas, assim a ferramenta Teleodontologia pode, além de auxiliar esse profissional em ações de educação permanente, permitir que consiga informações de local de referenciamento do paciente.

Pacheco et al. (2018) avaliaram o perfil da utilização dos serviços ofertados pelo Telessaúde no Estado do Espírito Santo de 2012 a 2017. Apesar da baixa participação no início, o programa tem se mostrado como importante instrumento de apoio técnico-assistencial. Os profissionais da equipe de saúde bucal vêm se cadastrando de maneira progressiva no programa, e esse aumento pode estar relacionado à oferta de atividades específicas de Odontologia, como teleconsultorias assíncrona e síncrona, webpalestras, biblioteca virtual, entre outras, e ao planejamento e desenvolvimento dos produtos a partir da necessidade da referida equipe cadastrada. Esse dado é semelhante aos do presente estudo, na medida em que, dos 230 cadastrados, foram obtidas 137 questões dos profissionais pertencentes a ESB, ou seja, é perceptível uma grande adesão dos profissionais da ESB.

A formação em Odontologia sempre esteve pautada prioritariamente no exercício privado da profissão, com ênfase no modelo biomédico e curativo, com cursos de Odontologia voltados para essa prática. Contudo, com o advento do SUS, as Diretrizes Curriculares Nacionais (DCN) tiveram mudanças também. Assim, a Teleodontologia, em consonância com as DCN, pode ampliar a inserção das Instituições de Ensino Superior (IES) nas ações de capacitação e educação permanente dos profissionais do SUS, fortalecendo a integração ensino-serviço (Haddad \& Skelton-Macedo, 2012; Caldarelli \& Haddad, 2016), afinal as respostas dos profissionais são pautadas em estudos e artigos científicos, além de recomendações vinculadas ao Ministério da Saúde.

Dentre as limitações do estudo está o fato de que no período analisado nem sempre o profissional teve acesso a um ponto de informática na sua Unidade de Saúde, visto que este processo foi gradativo.

\section{Conclusão}

Houve acesso gradativo do profissional cirurgião dentista a ferramenta do Telessaúde, contando em 2019 com 100\% das Unidades de Saúde com pontos de informática. Dentre as dúvidas mais prevalentes tem-se diagnóstico e uso de medicação, principalmente para grupos específicos como gestantes e hipertensos. Houve grande adesão pelo uso da ferramenta pelos profissionais auxiliando no processo de melhor atendimento à população e diagnósticos pautados em referencias do Ministério da Saúde.

\section{Agradecimentos}

À Fundação Estadual de Saúde - FUNESA pela disponibilidade dos dados.

\section{Referências}

Brasil. (2019a). Ministério da Saúde. Programa Brasil Sorridente.http://189.28.128.100/dab/docs/portaldab/publicacoes/acoes_politica_saude_bucal.pdf.

Brasil. (2019b). Ministério da Saúde. Programa Nacional Telessaúde Brasil Redes. http://www.saude.gov.br/trabalho-educacao-e-qualificacao/gestao-daeducacao/qualificacao-profissional/telessaude. 
Research, Society and Development, v. 10, n. 3, e29910313361, 2021

(CC BY 4.0) | ISSN 2525-3409 | DOI: http://dx.doi.org/10.33448/rsd-v10i3.13361

Brasil. (2019c). Ministério da Saúde. Programa Nacional Telessaúde Brasil Redes. http://www.saude.gov.br/trabalho-educacao-e-qualificacao/gestao-daeducacao/qualificacao-profissional/telessaude.

Brasil. Ministério da Educação. (2020a). Diretrizes Curriculares Nacionais do curso de graduação em Odontologia. http://portal.mec.gov.br/index.php?option=com_docman\&view=download\&alias=127951-pces803-18-1\&category_slug=outubro-2019\&Itemid=30192.

Brasil. Ministério da Saúde. (2020b). Informação e Gestão da Atenção Básica. https://egestorab.saude.gov.br/paginas/acessoPublico/rel atorios/relHistoricoCoberturaSB.xhtml.

Caldarelli, P. G., \& Haddad, A. E. (2016). Teleodontologia em consonância com as Diretrizes Curriculares Nacionais no desenvolvimento de competências profissionais. Rev $A B E N O, 16(2), 25-32$.

Correia, A. D., Dobashi, B. F., Gonçalves, C. C., Monreal, V. R., Nunes, E. A., Haddad, P. O., et al. (2014). Teleodontologia no programa nacional telessaúde Brasil redes: relato da experiência em Mato Grosso Do Sul. Rev ABENO, 14(1), 17-29.

DAB - Departamento de Atenção Básica. (2019). http://aps.saude.gov.br/.

FUNESA - Fundação Estadual de Saúde (2021). https://telessaude.se.gov.br/teleconsultoria/

Haddad, A. E., \& Skelton-Macedo, M. C. (2012). Teleodontologia na formação dos profissionais de saúde. In: Mathias, I., \& Monteiro A. (Org.). Gold book: Inovação tecnológica em educação e saúde. Rio de Janeiro: EdUERJ. http:// www.telessaude.uerj.br/resource/goldbook/pdf/12.pdf.

Jampani, N. D., Nutalapati, R., Dontula, B. S. K., \& Boyapati, R. (2011). Applications of teledentistry: A literature review and update. J Int Soc Prev Community Dent, 1(2), 37-44.

Narval, P. C. (2006). Saúde bucal coletiva: caminhos da odontologia sanitária à bucalidade. Rev Saúde Pública, 40, 141-147.

Pacheco, K. T. S., Nascimento, R. M., Rios, M. Z., Pacheco Filho, A. C., Barreira-Nielsen, C., \& Garbin, C. A. S. (2018). Tecnologias de informação e comunicação para formação profissional em Odontologia: uma proposta do Telessaúde/ES. Rev ABENO, 8(18), 127-136.

Sergipe. (2019). Secretaria de Estado da Saúde. Telessaúde Sergipe alcança 100\% de cobertura no estado. https://www.saude.se.gov.br/?p=30539. 